\title{
On the Unimodality of Independence Polynomials of Very Well-Covered Graphs
}

\author{
J. I. Brown* and B. Cameron \\ Department of Mathematics and Statistics \\ Dalhousie University, Halifax, NS B3H 3J5, Canada
}

\begin{abstract}
The independence polynomial $i(G, x)$ of a graph $G$ is the generating function of the numbers of independent sets of each size. A graph of order $n$ is very well-covered if every maximal independent set has size $n / 2$. Levit and Mandrescu conjectured that the independence polynomial of every very well-covered graph is unimodal (that is, the sequence of coefficients is nondecreasing, then nonincreasing). In this article we show that every graph is embeddable as an induced subgraph of a very well-covered graph whose independence polynomial is unimodal, by considering the location of the roots of such polynomials.
\end{abstract}

\section{Introduction}

A subset $S$ of the vertex set of a (finite, undirected) graph $G$ is said to be independent if $S$ induces a graph with no edges. The independence polynomial of a graph $G$ is defined to be

$$
i(G, x)=\sum_{k=0}^{\alpha} i_{k} x^{k},
$$

where $i_{k}$ is the number of independent sets of size $k$ in $G$ and $\alpha=\alpha(G)$, the independence number of $G$, is the size of the largest independent set in $G$. The independence polynomial is the generating function of the independence sequence $\left\langle i_{0}, i_{1}, \ldots, i_{\alpha}\right\rangle$. The independence polynomial of a graph has been of considerable interest [2, 3, 14 16,18 , $21-23$ since it was first defined by Gutman and Harary in 1983 as a generalization of the matching polynomial.

\footnotetext{
*Communicating author. Jason.Brown@dal.ca
} 
For many graph polynomials (such as matching [17], chromatic [19,27] and reliability [11,20] polynomials), the (absolute value of the) coefficient sequence, under a variety of bases expansions, have long been conjectured to be (or proven to be) unimodal, that is, nondecreasing then nonincreasing. We say that a polynomial is unimodal if its sequence of coefficients is unimodal.

What can we say about the unimodality of independence polynomials? They certainly form a sequence of positive integers. Alavi et al. [1] showed, in general, that the independence sequence $\left\langle i_{k}\right\rangle$ of a graph $G$ can be far from unimodal, for example, the graph $K_{25}+4 K_{2}$ has independence sequence $\langle 1,33,24,32,16\rangle$. More examples of graphs with nonunimodal independence sequences can be found in [1].

However, there are classes of graphs for which the independence coefficients are indeed unimodal. In a beautiful paper [10], Chudnovsky and Seymour provedthat the coefficients of the independence polynomials of claw-free graphs (that is, those without an induced star on 4 vertices) are unimodal.

Another highly structured family of graphs with respect to independence are wellcovered graphs, those whose maximal independent sets all have the same size (complete graphs and the 5-cycle are examples). The structure of such graphs has attracted considerable attention in the literature, with characterizations for those of high girth [13]. In [6], the authors conjectured that the independence coefficients of well-covered graphs were unimodal, and showed that every graph $G$ can be embedded as an induced subgraph of such a well-covered graph. However, Michael and Traves [26] later disproved the conjecture. A conjecture due to Alavi et al. [1] that is still open is that the independence polynomial of a tree is unimodal.

Finally, Levit and Mandrescu [24] amended the original unimodality conjecture on well-covered graphs as follows. A very well-covered graph $G$ of order $n$ (that is, on $n$ vertices) is a well-covered graph for which every maximal independent set has size $n / 2$; for example, the complete bipartite graphs $K_{m, m}$ are very well-covered. Other examples are afforded by the following construction. Let $G$ be any graph. Form $G^{*}$, the leafy extension of $G$ (sometimes also called the corona of $G$ with $K_{1}$ ) from $G$ by attaching, for each vertex $v$ of $G$ a new vertex $v^{*}$ to $v$ with an edge (such a vertex is called a pendant vertex); leafy extensions are always very well-covered (more about that shortly).

Levit and Mandrescu conjectured that the coefficients of the independence polynomials of a very well-covered graph are unimodal, and to date, the conjecture remains open. Some partial results have been proven on the tail of independence sequences of very well-covered graphs [25] and the first $\left\lceil\frac{\alpha}{2}\right\rceil$ terms have been shown to be nondecreasing for well-covered graphs [26]. The conjecture is known to hold when $\alpha(G) \leq 9[25$ and for leafy extensions of any graph $G$ where $\alpha(G) \leq 8[9]$, or where $G$ is a path or star [22]. In this paper we shall show that Levit and Mandrescu's conjecture holds for some iterated leafy extensions of any graph $G$. 


\section{Unimodality of Independence Polynomials of Leafy Extensions and Sectors in the Complex Plane}

The leafy extension $G^{*}$ of any graph $G=(V, E)$ of order $n$ is always very-well-covered. Clearly, $\alpha\left(G^{*}\right) \leq n$, as the graph has a perfect matching (and no independent set can contain two vertices that are matched). Moreover, $\alpha\left(G^{*}\right)=n$ as any independent set $I$ of $G$ can be extended to one in $G^{*}$ by adding in any subset of $(V-I)^{*}=\left\{v^{*}: v \in V-I\right\}$. It follows (see also 22]) that if $i(G, x)=\sum i_{k} x^{k}$, then

$$
\begin{aligned}
i\left(G^{*}, x\right) & =\sum i_{k} x^{k}(1+x)^{n-k} \\
& =(1+x)^{n} \cdot i\left(G, \frac{x}{1+x}\right) .
\end{aligned}
$$

For a graph $G$ and positive integer $k$, let $G^{k *}$ denote the $k$-th iterated leafy extension of $G$, that is, the graph formed by recursively attaching pendant vertices, $k$ times:

$$
G^{k *}= \begin{cases}G^{*} & \text { if } k=1 \\ \left(G^{(k-1) *}\right)^{*} & \text { if } k \geq 2\end{cases}
$$

Figure 2.1 shows the graph $P_{4}^{2 *}$.

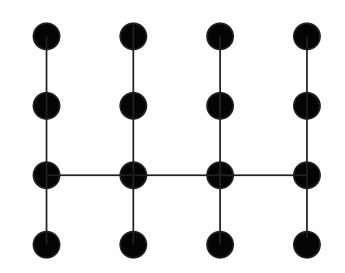

Figure 2.1: The double leafy extension of $P_{4}$.

We can extending formula (1) to higher iterations of the $*$ operation as follows.

Proposition 2.1. For any graph $G$ of order $n$ and any positive integer $k$,

$$
i\left(G^{k *}, x\right)=i\left(G, \frac{x}{k x+1}\right)(k x+1)^{n} \prod_{\ell=1}^{k-1}(\ell x+1)^{n 2^{k-\ell-1}} .
$$

Proof. We proceed by induction on $k$, the number of iterations of the $*$ operation. The base case follows directly from (1), so we can assume that the result holds for some $k \geq 1$, i.e.,

$$
i\left(G^{k *}, x\right)=i\left(G, \frac{x}{k x+1}\right)(k x+1)^{n} \prod_{\ell=1}^{k-1}(\ell x+1)^{n 2^{k-\ell-1}} .
$$


A trivial induction shows that $G^{k *}$ has order $n 2^{k}$. From these, the fact that $G^{(k+1) *}=$ $\left(G^{k *}\right)^{*}$ and formula (1) we derive that

$$
\begin{aligned}
i\left(G^{(k+1) *}, x\right) & =(1+x)^{n 2^{k}} i\left(G^{k *}, \frac{x}{x+1}\right) \\
& =(1+x)^{n 2^{k}} i\left(G, \frac{\frac{x}{x+1}}{\frac{k x}{x+1}+1}\right)\left(k\left(\frac{x}{x+1}\right)+1\right)^{n} \prod_{\ell=1}^{k-1}\left(\ell\left(\frac{x}{x+1}\right)+1\right)^{n 2^{k-\ell-1}} \\
& =(1+x)^{n 2^{k}} i\left(G, \frac{x}{(k+1) x+1}\right)\left(\frac{(k+1) x+1}{x+1}\right)^{n} \prod_{\ell=1}^{k-1}\left(\frac{(\ell+1) x+1}{x+1}\right)^{n 2^{k-\ell-1}} \\
& =\frac{(1+x)^{n 2^{k}}}{(1+x)^{n 2^{k-1}}} i\left(G, \frac{x}{(k+1) x+1}\right)((k+1) x+1)^{n} \prod_{\ell=1}^{k-1}((\ell+1) x+1)^{n 2^{k-\ell-1}} \\
& =(1+x)^{n 2^{k-1}} i\left(G, \frac{x}{(k+1) x+1}\right)((k+1) x+1)^{n} \prod_{\ell=1}^{k-1}((\ell+1) x+1)^{n 2^{k-\ell-1}} \\
& =i\left(G, \frac{x}{(k+1) x+1}\right)((k+1) x+1)^{n} \prod_{\ell=0}^{k-1}((\ell+1) x+1)^{n 2^{k-\ell-1}} \\
& =i\left(G, \frac{x}{(k+1) x+1}\right)((k+1) x+1)^{n} \prod_{\ell=1}^{k}(\ell x+1)^{n 2^{(k+1)-\ell-1}} .
\end{aligned}
$$

There are many techniques for proving that a sequence is unimodal (see for example, [28] and [4]). One that has been frequently applied is due to Newton (c.f. [12, pp. 270271]), who proved that if a polynomial $p(x)=a_{0}+a_{1} x+\cdots+a_{n} x^{n}$ with positive coefficients has all real roots, then the sequence $\left\langle a_{0}, a_{1}, \ldots, a_{n}\right\rangle$ satisfies

$$
a_{i}^{2} \geq \frac{i+1}{i} \frac{n-i+1}{n-i} a_{i-1} a_{i+1}
$$

and hence is $\log$ concave, that is $a_{i}^{2} \geq a_{i-1} a_{i+1}$ for all $0<i<n$ (in fact, the sequence is strictly $\log$ concave as $a_{i}^{2}>\frac{i+1}{i} \frac{n-i+1}{n-i} a_{i-1} a_{i+1}$ holds for all relevant $i$ ). In such a case, it follows directly that the sequence is unimodal as well. Newton's simple but elegant theorem has been used to prove that a variety of sequences (and polynomials) are unimodal, such as matching polynomials [17 and the independence polynomials of claw-free graphs $[10]$.

From Proposition 2.1 we see at once that all independence roots of $G$ are real if and only if the same is true of its leafy extension. As most independence polynomials have a non-real root [8], we won't be able to get at our desired result, namely that for any graph $G$ the independence polynomial of some iterated leafy extension of $G$ is unimodal, via Newton's theorem. 
However, Newton's theorem is only a sufficient condition for the coefficient sequence to be log concave. Brenti et al. [5] weakened the conditions as follows:

Proposition 2.2 ( [5]). If all the roots $z$ of the polynomial $f(x) \in \mathbb{R}[x]$ are in the region

$$
\left\{z \in \mathbb{C}:|\arg (z)|<\frac{\pi}{3}\right\},
$$

then the sequence of coefficients of $f(x)$ is strictly log concave and alternates in sign.

Replacing $f(x)$ by $f(-x)$, we derive that:

Corollary 2.3. If all the roots $z$ of the polynomial $f(x) \in \mathbb{R}[x]$ are in the region

$$
\left\{z \in \mathbb{C}: \frac{2 \pi}{3}<|\arg (z)|<\frac{4 \pi}{3}\right\}
$$

then the sequence of coefficients of $f(x)$ is strictly log concave (and the sequence of coefficients of $f(x)$ is either all positive or all negative).

We shall make good use out of this corollary now to prove our main result on log concavity of independence polynomials of leafy extensions, via an excursion through their roots.

Theorem 2.4. For all graphs $G$ let

$$
M=\max \left\{\frac{\frac{1}{\sqrt{3}}|\operatorname{Im} z|+|\operatorname{Re} z|}{|z|^{2}}: z \text { is a root of } I(G, x)\right\} .
$$

If $k>M$, then the coefficients of $I\left(G^{k *}, x\right)$ are strictly log concave.

Proof. From Proposition 2.1 it follows that if $r_{1}, \ldots, r_{m}$ are the roots of $I(G, x)$, then the roots of $I\left(G^{k *}, x\right)$ are $\frac{r_{i}}{1-k r_{i}}$ for $i=1,2, \ldots, m$ along with the rational numbers $\frac{-1}{\ell}$ for $\ell=1,2, \ldots, k$. Let $r$ be any root of $I(G, x)$, and set $a=\operatorname{Re} r$ and $b=\operatorname{Im} r$. Note that either $a$ or $b$ is nonzero since 0 is not the root of any independence polynomial and likewise, $r \neq 1 / k$ for all $k \geq 0$. We expand a root of $I\left(G^{k *}, x\right)$ to obtain,

$$
\begin{aligned}
\frac{r}{1-k r} & =\frac{a+i b}{1-k(a+i b)} \\
& =\frac{a+i b}{(1-k a)-i k b} \cdot \frac{(1-k a)+i k b}{(1-k a)+i k b} \\
& =\frac{a(1-k a)+i a k b+i b(1-k a)-k b^{2}}{(1-k a)^{2}+k^{2} b^{2}} \\
& =\frac{a(1-k a)-k b^{2}+i(a k b+b(1-k a))}{(1-k a)^{2}+k^{2} b^{2}} \\
& =\frac{\left(a-k a^{2}-k b^{2}\right)+i b}{(1-k a)^{2}+k^{2} b^{2}} .
\end{aligned}
$$


We now wish to show that for sufficiently large $k$, the root $z=\frac{r}{1-k r}$ of $I\left(G^{k *}, x\right)$ lies in the sector $\left\{z \in \mathbb{C}: \frac{2 \pi}{3}<|\arg (z)|<\frac{4 \pi}{3}\right\}$; the result will then follow immediately from Corollary 2.3 (as the negative rational roots obviously lie in the sector). It is clear to see that $z$ lies in the sector if and only if $\operatorname{Re} z<0$ and $\left|\frac{\operatorname{Im} z}{\operatorname{Re} z}\right|<\sqrt{3}$. Now, $\operatorname{Re} z=\frac{a-k a^{2}-k b^{2}}{(1-k a)^{2}+k^{2} b^{2}}$ and $(1-k a)^{2}+k^{2} b^{2}>0$ since if $b=0$ then $a \neq 1 / k$. We also have $a-k a^{2}-k b^{2}=-k\left(a^{2}+b^{2}\right)+a$ and so for $k>\frac{(1 / \sqrt{3})|b|+|a|}{a^{2}+b^{2}} \geq \frac{|a|}{a^{2}+b^{2}}$, it follows that Re $z<0$. We note as well that for $\left.k>\frac{|a|}{a^{2}+b^{2}}, k\left(a^{2}+b^{2}\right)-a\right)$ is positive and increasing, as a function of $k$, and that $\frac{(1 / \sqrt{3})|b|+|a|}{a^{2}+b^{2}} \geq \frac{|a|}{a^{2}+b^{2}}$. We now compute the ratio of the imaginary and real part of $z$ :

$$
\begin{aligned}
\left|\frac{\operatorname{Im} z}{\operatorname{Re} z}\right| & =\left|\frac{b}{k\left(a^{2}+b^{2}\right)-a}\right| \\
& <\frac{|b|}{\left|\left(\frac{(1 / \sqrt{3})|b|+|a|}{a^{2}+b^{2}}\right)\left(a^{2}+b^{2}\right)-a\right|} \\
& =\frac{|b|}{(1 / \sqrt{3})|b|+|a|-a} \\
& \leq \sqrt{3} .
\end{aligned}
$$

The result now follows from Corollary 2.3 .

Corollary 2.5. Every graph $G$ on $n$ vertices is an induced subgraph of a very wellcovered graph $H$ such that the sequence of coefficients of $I(H, x)$ is unimodal.

\section{Concluding Remarks}

While Theorem 2.4 shows that for any graph $G$, the independence polynomial of some iterated leafy extension of $G$ is unimodal, the question remains as to whether this is true for every iterated leafy extension, and, in particular, for the leafy extension of $G$. From the properties of linear fractional transformations, we can explicitly state where the independence roots of $G$ need to lie to ensure its leafy extension has a log concave (and hence unimodal) independence polynomial.

Theorem 3.1. If the roots of $I(G, x)$ lie outside of the region bounded by the union of circles with with radii $\frac{\sqrt{3}}{3}$ centred at $\frac{1}{2}+\frac{\sqrt{3} i}{6}$ and $\frac{1}{2}-\frac{\sqrt{3} i}{6}$, then the $i\left(G^{*}, x\right)$ is strictly log-concave.

Proof. We will find the image of the region $R=\left\{z \in \mathbb{C}: \frac{2 \pi}{3}<|\arg (z)|<\frac{4 \pi}{3}\right\}$ under the Möbius transformation $f(z)=\frac{z}{1+z}$. Such a transformation sends lines and circles to 
lines and circles, and interiors/exteriors of circles and half-planes are sent to the same set of regions. We need only find the image of three points on the two line segments bounding the sector. The images of $-1+\sqrt{3} i, 0$, and $\infty$ are $1+\frac{\sqrt{3} i}{3}, 0$, and 1 respectively, yielding the circle $C_{1}$, centred at $\frac{1}{2}+\frac{\sqrt{3} i}{6}$ with radius $\frac{\sqrt{3}}{3}$. As $1 / 2$ is inside $C_{1}$ and gets mapped to $\frac{1}{3}$, which is above the $\operatorname{line} \arg (z)=\frac{2 \pi}{3}$, the exterior of $C_{1}$ gets mapped below the line $\arg (z)=\frac{2 \pi}{3}$.

Similarly, the images of $-1-\sqrt{3} i, 0$, and $-\infty$ are $1-\frac{\sqrt{3} i}{3}, 0$, and 1 respectively, yielding the circle $C_{2}$, centred at $\frac{1}{2}-\frac{\sqrt{3} i}{6}$ with radius $\frac{\sqrt{3}}{3}$. As $1 / 2$ is inside $C_{2}$ and is mapped to $\frac{1}{3}$ which is below the line $\arg (z)=\frac{4 \pi}{3}$, the exterior of $C_{2}$ gets mapped below the line $\arg (z)=\frac{4 \pi}{3}$. Therefore, if we take an exterior point to the union of $C_{1}$ and $C_{2}$ is must have image under $f$ above the line $\arg (z)=\frac{4 \pi}{3}$ and below the line $\arg (z)=\frac{2 \pi}{3}$, i.e., in the region $R$. Therefore, by Corollary $2.3, I\left(G^{*}, x\right)$ is strictly log-concave.

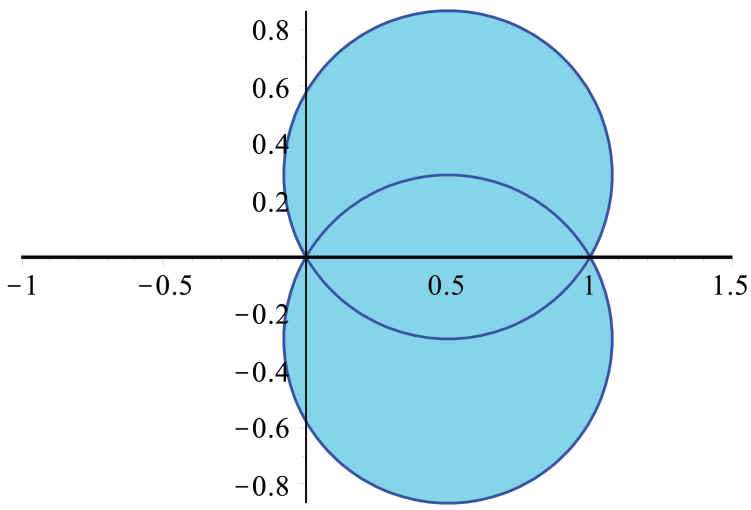

Figure 3.1: Region that ends up outside the sector $R=\left\{z \in \mathbb{C}: \frac{2 \pi}{3} \leq|\arg (z)|<\frac{4 \pi}{3}\right\}$ under the Möbius transformation $f(z)=\frac{z}{1+z}$.

Theorem 3.1 assures us that as long as the roots of $I(G, x)$ are outside of a region in $\mathbb{C}$ with area a little more than 2 , then $I\left(G^{*}, x\right)$ will be strictly log-concave. Although this result works for many graphs, Brown, Hickman, and Nowakowski [7| showed that the set of independence roots of all graphs is dense in $\mathbb{C}$ even when restricted to wellcovered graphs. Therefore, there exist graphs with independence roots in the union of the interior of the two circles specified in the statement of Theorem 3.1 and therefore graphs that have leafy extensions with independence roots outside of $R$. In fact, using the methods outlined in [7] to find independence roots throughout $\mathbb{C}$, we have found that $I\left(G^{*}, x\right)$ has a root outside of $R$ for $G=P_{5}\left[\overline{K_{6}}\right]$ and $G=P_{6}\left[\overline{K_{11}}\right]$ (here $G[H]$ is the graph formed from $G$ by substituting a copy of $H$ in for each vertex of $G$ - this is sometimes known as the lexicographic product of $G$ with $H$ ), although the independence polynomials in these cases turn out to be log-concave as well. 
Another point to note is that there exist graphs that are very well-covered but are not the leafy extension of another graph: some examples which were already pointed out are the bipartite graphs $K_{n, n}$ among others. Our results do not encompass these results; however, Finbow et al. [13] showed that, with the exceptions of $K_{1}$ and $C_{7}$, a graph $G$ with girth $(G) \geq 6$ is well-covered if and only if its pendant edges form a perfect matching. It is easy to see that the pendant edges of $G$ forming a perfect matching is equivalent to $G=H^{*}$ for some graph $H$ and therefore, our results apply to every well-covered graph with girth at least 6 .

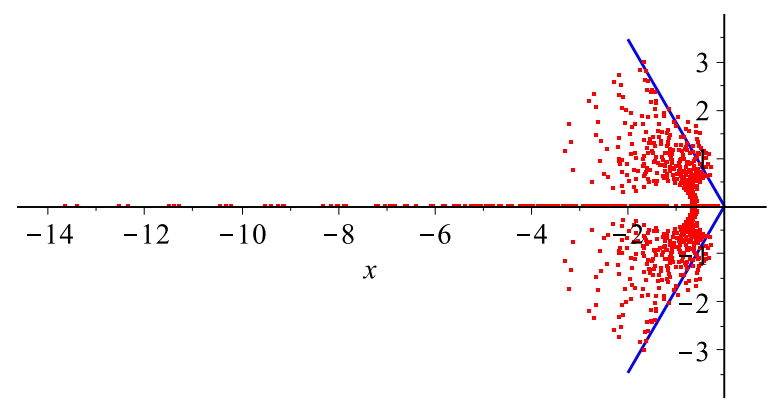

Figure 3.2: Independence roots of all connected graphs of order 8 .

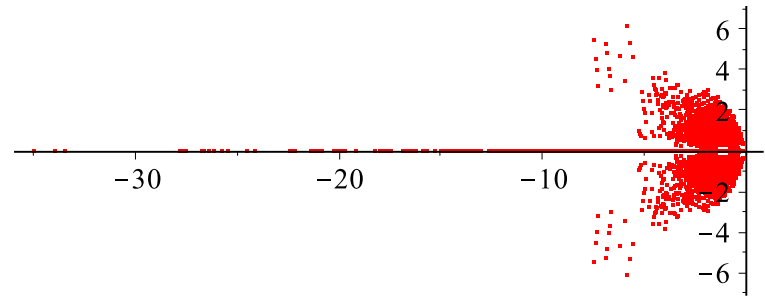

Figure 3.3: Independence roots of all trees of order 14.

Finally, the distribution of independence roots with respect to the sector

$$
\left\{z \in \mathbb{C}: \frac{2 \pi}{3}<|\arg (z)|<\frac{4 \pi}{3}\right\}
$$

is a fascinating one. Some computations suggest that most small graphs have their roots in the sector; in fact, out of 11, 117 connected graphs of order 8, there are only 40 independence roots (counting multiplicities) outside the sector (see Figure 3.2). Hence we propose the following:

Conjecture 3.2. The independence polynomial of almost every graph $G$ of order $n$ has all of its roots in the sector $\left\{z \in \mathbb{C}: \frac{2 \pi}{3}<|\arg (z)|<\frac{4 \pi}{3}\right\}$, and hence is log concave. 
The same seems to be true of trees as well, and in fact we have not been able to find a single independence root of a tree in the right half-plane (see Figure 3.3). so we also conjecture the following:

Conjecture 3.3. The independence polynomials of trees $G$ are stable, that is, their roots lie in the left half-plane.

Acknowledgements: The first author would like to acknowledge the support of the Natural Sciences and Engineering Research Council of Canada (grant number 170450-2013).

\section{References}

[1] Y. Alavi, P. J. Malde, A. J. Schwenk, and P. Erdös. The vertex sequence of a graph is not constrained. Congressus Numer., 1987.

[2] G. E.; Andrews, R.; Askey, and R. Roy. Special functions, Encyclopedia of Mathematics and its Applications 7. Cambridge University Press, 2000.

[3] J. L. Arocha. Propriedades del polinomio independiente de un grafo. Revista Ciencias Matematicas, V:103-110, 1984.

[4] F. Brenti. Log-concave and unimodal sequences in algebra, combinatorics, and geometry: an update, in Jerusalem Combinatorics 93. Contemporary Mathematics, 178:71-89, 1994.

[5] F. Brenti, G. F. Royle, and D. G. Wagner. Location of zeros of chromatic and related polynomials of graphs. Canad. J. Math, 46:55-80, 1994.

[6] J. I. Brown, K. Dilcher, and R. J. Nowakowski. Roots of independence polynomials of well-covered graphs. J. Algebraic Combin., 11:197-210, 2000.

[7] J. I. Brown, C. A. Hickman, and R. J. Nowakowski. On the location of the roots of independence polynomials. Journal of Algebraic Combinatorics, 19:273-282, 2004.

[8] J. I. Brown and R. J. Nowakowski. Avergae Independence Polynomials. J. Comb. Th. B, 93:313-318, 2005.

[9] S.-Y. Chen and H.-J. Wang. Unimodality of independence polynomials of very well-covered graphs. Ars Combin., 97A:509-529, 2010.

[10] Maria Chudnovsky and Paul Seymour. The roots of the independence polynomial of a clawfree graph. Journal of Combinatorial Theory, Series B, 97(3):350-357, 2007. 
[11] C.J. Colbourn. The Combinatorics of Network Reliability. Oxford University Press, Oxford and New York, 1987.

[12] L. Comtet. Advanced Combinatorics. Reidel, Boston, 1974.

[13] A.; Finbow, B; Hartnell, and R. J. Nowakowski. A characterization of well covered graphs of girth 5 or greater. J. Combin. Th. B, 57:44-68, 1993.

[14] I. Gutman. Some relations for the independence and matching polynomials and their chemical applications. Bul. Acad. Serbe Sci. arts, 105:39-49, 1992.

[15] I. Gutman and F. Harary. Generalizations of the matching polynomial. Utilitas Mathematica, 24:97-106, 1983.

[16] I. Gutman and X. Li. A unified approach to the first derivatives of graph polynomials. Discrete Applied Mathematics, 58:293-297., 1995.

[17] O. J. Heilmann and E. H. Lieb. Theory of monomer-dimer systems. Communications in Mathematical Physics, 25(3):190-232, 1972.

[18] C. Hoede and X. Li. Clique polynomials and independent set polynomials of graphs. Discrete Mathematics, 125:219-228, 1994.

[19] June Huh. Milnor numbers of projective hypersurfaces and the chromatic polynomial of a graph. J. Amer. Math. Soc., 25:907-927, 2012.

[20] June Huh. h-Vectors of matroids and logarithmic concavity. Advances in mathematics, 270:49-59, 2015.

[21] V. E. Levit and Eugen Mandrescu. On well-covered trees with unimodal independence polynomials. Congressus Numerantium, 159:193-202, 2002.

[22] V. E. Levit and Eugen Mandrescu. Very well-covered graphs with log-concave independence polynomials. Carpathian Journal of Mathematics, 20:73-80, 2004.

[23] V. E. Levit and Eugen Mandrescu. The independence polynomial of a graph-a survey. Proceedings of the 1st International Conference on Algebraic Informatics, $\mathrm{V}: 233-254,2005$.

[24] V. E. Levit and Eugen Mandrescu. Independence polynomials of well-covered graphs: generic counterexamples for the unimodality conjecture. Europ. J. Combin, 27:931-939, 2006.

[25] V. E. Levit and Eugen Mandrescu. Independence polynomials and the unimodality conjecture for very well-covered, quasi-regularizable, and perfect graphs. In Graph Theory in Paris Proceedings of a Conference in Memory of Claude Berge, pages 243-254, 2007. 
[26] T. S. Michael and W. N. Traves. Independence sequences of well-covered graphs: non-unimodality and the roller-coaster conjecture. Graphs and Combin., 19:403411, 2003.

[27] R. C. Read. An introduction to chromatic polynomials. Journal of Combinatorial Theory, 4:52-71, 1968.

[28] R. P. Stanley. Log-concave and unimodal sequences in algebra, combinatorics, and geometry. Annals of the New York Academy of Sciences, 576:500-535, 1989. 